\title{
Cluster algebras in scattering amplitudes with special 2D kinematics
}

\author{
Marcus A. C. Torres $^{\mathrm{a}}$ \\ Institut de Physique Théorique, CEA-Saclay, 91191 Gif-sur-Yvette Cedex, France
}

Received: 30 October 2013 / Accepted: 27 January 2014 / Published online: 27 February 2014

(c) The Author(s) 2014. This article is published with open access at Springerlink.com

\begin{abstract}
We study the cluster algebra of the kinematic configuration space $\operatorname{Conf}_{n}\left(\mathbb{P}^{3}\right)$ of an $n$-particle scattering amplitude restricted to the special 2D kinematics. We found that the $n$-point two-loop MHV remainder function in special 2D kinematics depends on a selection of the $\mathcal{X}$-coordinates that are part of a special structure of the cluster algebra related to snake triangulations of polygons. This structure forms a necklace of hypercube beads in the corresponding Stasheff polytope. Furthermore at $n=12$, the cluster algebra and the selection of the $\mathcal{X}$-coordinates in special 2D kinematics replicates the cluster algebra and the selection of $\mathcal{X}$-coordinates of the $n=6$ two-loop MHV amplitude in 4D kinematics.
\end{abstract}

\section{Contents}

1 Introduction . . . . . . . . . . . . 1

2 Special 2D kinematics . . . . . . . . . . . . 2

3 Cluster algebras and Stasheff polytopes . . . . . . 3

4 Configuration space and its cluster structure . . . . 4

5 Results for $n=8,10$, and $12 \ldots . . . . .66$

$5.1 n=8 \ldots \ldots \ldots$

$5.2 n=10 \ldots \ldots \ldots$

$5.3 n=12 \ldots \ldots \ldots 6$

6 General $n$ external particles . . . . . . . . . . 8

7 Conclusion ................ . . 9

References............... 10

\section{Introduction}

$\mathcal{N}=4$ supersymmetric Yang-Mills in its planar limit is the present arena for developing new methods of calculating scattering amplitudes of perturbative QCD. Feynman diagrams revealed itself to be cumbersome and incapable of unveiling the beautiful symmetries and structures of $N=4$ SYM.

\footnotetext{
a e-mail: marcus-andre.de-carvalho-torres@cea.fr
}

Among these symmetries, it was realized [1] that $\mathcal{N}=4$ SYM at tree level is superconformal in Minkowski space and in the dual space and at any loop order one can calculate amplitudes by calculating Wilson loops in the dual space, which led to several results [2-9].

One of the present challenges of $N=4$ SYM is that amplitudes at L-loops involve complicated polylogarithm functions of transcendental type 2L. Such functions have several interrelations and an amplitude can be written in different forms. Also there are numerous choices for the conformal cross ratios as arguments.

In $[10,11]$, the authors Golden, Goncharov, Spradlin, Vergu and Volovich (GGSVV) showed that a judicious choice of kinematic variables was one of the main ingredients in a great simplification of the previously calculated two-loop six-particle MHV remainder function $\mathcal{R}_{n}^{(2)}$ [12-14] and that this choice is related to the cluster structure that is intrinsic to the kinematic configuration space $\operatorname{Conf}_{n}\left(\mathbb{P}^{3}\right)$ of the external particles. Its cluster structure selects the appropriate cross ratios (directly related to $\mathcal{X}$-coordinates in the cluster algebras) to be used in the motivic amplitudes [11] and intriguingly some cluster algebras define arguments so suitably to some polylogarithmic functional equations like in the famous Abel's pentagon dilogarithm identity and in a recently found trilogarithm relation [11]. All this shows that the use of cluster coordinates as arguments in remainder functions may be the appropriate way to simplify the long logarithm expressions. Fortunately, this cluster structure, which belongs to the kinematic configuration space, has been shown [11] to be preserved at two-loop order where the polylogarithmic identities of weight four are less known. Other interesting properties of the cluster structure of the configuration space, such as positivity and the fact that the logarithms of their $\mathcal{X}$ coordinates are canonical Darboux coordinates of a Poisson space [15], led the authors of [11] to propose that the variables which should appear in the motivic MHV amplitudes in SYM theory are cluster $\mathcal{X}$-coordinates of the cluster algebra of its configuration space. 
The use of the cluster structure of $\operatorname{Conf}_{n}\left(\mathbb{P}^{3}\right)$ worked efficiently for $n=6,7$, but for $n>7$ the cluster algebra is of infinite type and we cannot count all of its $\mathcal{A}$-coordinates, $\mathcal{X}$-coordinates, and clusters. In order to overcome such a problem, here we focus on the study of finite cluster subalgebras for $n \geq 8$ by reducing the configuration space to a certain kinematic limit.

In special 2D kinematics, the cluster sub-algebras are always finite and in this case the algebra can be related to a Lie Algebra with a simply laced Dynkin diagram [16]. Also the two-loop MHV remainder function in special 2D kinematics has been fully calculated at $n$ larger than 7 [17], and we are able to check the existence of the cluster structure in amplitudes in this kinematic domain.

The program of research initiated by [11] is a very instigating one and many questions remain to be answered. For example, at $n=6$ and at $n=7$ only $3 / 5$ of all cluster $\mathcal{X}$-coordinates of their respective cluster algebra show up in the two-loop MHV motivic amplitude. Another question is that present studies of cluster algebras are done concerning results on two-loop MHV amplitudes only [18], but possibly some of the cluster structure of the configuration space is preserved at higher loops and non-MHV amplitudes.

In this regard, a small drawback exists in special 2D kinematics concerning its cluster structure. At $n=8, \mathrm{~N}^{2} \mathrm{MHV}$ one-loop, NMHV two-loop and MHV three-loop amplitudes (remainder functions) were calculated in [19] and these results were found to have the symbols, polylogarithm arguments and poles with mixing $\mathcal{X}$-coordinates $\mathrm{v}$ and $\mathrm{w}$ of its $A_{1} \times A_{1}$ cluster algebra. The mixing of $\mathcal{X}$-coordinates occurs in the form of $v-w$ and $1-v w$. This structure is believed to exist in 2D special kinematics for $k+l \geq 3 \mathrm{~N}^{k}$ MHV l-loop amplitudes. Nevertheless, the cluster structure continues to have a role in the selection of cross ratios ( $v$ and $w$ ). The different structure of the arrangement of $\mathcal{X}$-coordinates in the arguments of the polylogarithm expressions in special 2D kinematics compared with two-loop MHV amplitudes in 4D kinematics may be connected with the fact that the positivity condition ${ }^{1}$ of cluster $\mathcal{A}$-coordinates in special 2D kinematics and 4D kinematics are not the same, while the amplitude in special 2D kinematics is a reduction from the amplitude in 4D kinematics.

In addressing the study of a smaller configuration space we were also able to recognize that not all $\mathcal{X}$-coordinates appear in the remainder function and we were able to identify their main characteristics within the cluster structure, through the use of associated polygon triangulations and Stasheff polytopes. We were able to find in special 2D kinematics, at $n=12$, a double copy of the same $A_{3}$ cluster algebra as appears in the configuration space of $n=6$ in 4D kinemat-

${ }^{1}$ Observed by Song He, private communication. ics and equally the same nine out of 15 (for each copy) of the $\mathcal{X}$-coordinates appear in the remainder function. These nine $\mathcal{X}$-coordinates are sorted as $\mathcal{X}$-coordinates of the six snake clusters of the $A_{3}$ cluster algebra. We call 'snake clusters' the ones whose associated polygon triangulation has their diagonals associated to negative simple roots [20]. Such triangulations have a zig-zag or snake appearance, whence the name. We notice that snake clusters are part of a structure in their corresponding Stasheff polytope. We call it the 'necklace' of hypercube beads, or 'hypercube necklace'. In this structure (necklace) in the Stasheff polytope, the nearest snake cluster vertices are opposite vertices of a hypercube that connects them. All quadrilateral faces $\left(A_{1} \times A_{1}\right.$ cluster sub-algebras) in these hypercubes have its two $\mathcal{X}$-coordinates belonging to snake clusters. These snake clusters are the two which correspond to the vertices that connect the hypercube to the necklace in the Stasheff polytope.

In [11], the authors related obstruction terms in the amplitude expression to quadrilateral faces in the Stasheff polytope. Such terms are obstructions to write the two-loop MHV motivic amplitude in terms of classical 4-logarithms. In special 2D kinematics, such motivic amplitudes are trivial since the coproduct of a product of logarithms is trivial. Consequently, obstructions cannot be studied here but the cluster algebra that we study here is present in 4D kinematics where quadrilateral faces with selected snake cluster $\mathcal{X}$-coordinates may play such a role.

Our hope is that a similar study of the $\mathcal{X}$-coordinates may exist for type $E_{6}$ cluster algebras or its cluster sub-algebras, which may help justify in the case that $n=7$ [11] why only $3 / 5$ of all $\mathcal{X}$-coordinates of $E_{6}$ cluster algebra are used in the amplitude and why the obstruction terms of only 42 quadrilateral faces out of 1,785 quadrilateral faces existent in an $E_{6}$ Stasheff polytope are used.

This present work is organized as follows. In Sect. 2, we present special 2D kinematics [17,21] and cross ratios. In Sect. 3, we review the elements of the cluster algebra. In Sect. 4, we reduce the configuration space to special 2D kinematics and find its cluster algebra, its cluster coordinates, and the mutation relations. In Sect. 5, we study the cases for $n=8,10$, and 12 . The cases $n=8$ and 10 show the presence of the cluster structure of the configuration space among the arguments (cross ratios) of $\mathcal{R}_{n}^{(2)}$ in special 2D kinematics, while at $n=12$, we notice the relevant structure related to a selection of $\mathcal{X}$-coordinates by the amplitude which is further studied in the general case of $n$ external particles in Sect. 6 . We conclude in Sect. 7.

\section{Special 2D kinematics}

A good description of special 2D kinematics is presented in $[17,21]$ and we review a few aspects below. 
The special two-dimensional kinematics is the condition when all external particles have their momenta lying in the same $1+1$ dimensions while the internal particles propagate in $3+1$ dimensions. Due to energy-momentum conservation, the external momenta $p_{i}$ define a polygon in the dual space $x^{\mu}$

$p_{i}^{\mu}=x_{i}^{\mu}-x_{i+1}^{\mu}$.

The edges of the polygon, $p_{i}$, in a zig-zag way invert the spatial direction, i.e., they switch between light cone directions $x^{+}$and $x^{-}$[22] and this condition implies that there must be an even number of external particles.

As a result, the external momenta of particles in the light cone $(+,-)$ directions are:

$p_{i}= \begin{cases}\left(p_{i}^{+}, 0\right), & i \text { even } \\ \left(0, p_{i}^{-}\right), & i \text { odd }\end{cases}$

In the language of momentum twistors, it translates into

$Z_{i}= \begin{cases}\left(Z_{i}^{1}, 0, Z_{i}^{3}, 0\right) & i \text { even } \\ \left(0, Z_{i}^{2}, 0, Z_{i}^{4}\right) & i \text { odd }\end{cases}$

reducing $S L(4) \rightarrow S L(2)_{+} \times S L(2)_{-}$in $2 \mathrm{~d}$. The $S L(4)$ invariant product of four momentum twistors,

$\langle i j k l\rangle=\epsilon_{a b c d} Z_{i}^{a} Z_{j}^{b} Z_{k}^{c} Z_{l}^{d}$,

becomes zero, unless there are two odd and two even indices. In this case the even and odd indices factorize into $S L(2)_{ \pm}$ invariant terms. For example,

$\langle 1234\rangle=\langle 13\rangle\langle 24\rangle=\left(Z_{1}^{2} Z_{3}^{4}-Z_{1}^{4} Z_{3}^{2}\right)\left(Z_{2}^{1} Z_{4}^{3}-Z_{2}^{3} Z_{4}^{1}\right)$.

Writing the Lorenz invariant square distances of the dual coordinates in terms of the momentum twistors [23]:

$\left(x_{i j}\right)^{2}=\left(x_{i}-x_{j}\right)^{2}=\frac{\langle i i+1 j j+1\rangle}{\left\langle\lambda_{i} \lambda_{i+1}\right\rangle\left\langle\lambda_{j} \lambda_{j+1}\right\rangle}$

and applying it in a standard basis of cross ratios in fourdimensional kinematics [17]

$$
\begin{aligned}
u_{i j} & =\frac{x_{i j+1}^{2} x_{i+1 j}^{2}}{x_{i j}^{2} x_{i+1 j+1}^{2}} \\
& = \begin{cases}1 & i \text { and } \mathrm{j} \text { with opposite parity } \\
\frac{\langle i j+2\rangle\langle i+2 j\rangle}{\langle i j\rangle\langle i+2 j+2\rangle} & i \text { and } \mathrm{j} \text { with same parity }\end{cases}
\end{aligned}
$$

Therefore, in 2D kinematics the cross ratios are separated into two groups: those that depend only on momentum twistors with even indices and those that depend only on momentum twistors with odd indices.
In order to keep the notation in agreement with [17], we rewrite the above $2 \mathrm{D}$ cross ratios as

$u_{i j}^{+}:=\frac{\langle 2 i+1,2 j+3\rangle\langle 2 i+3,2 j+1\rangle}{\langle 2 i+1,2 j+1\rangle\langle 2 i+3,2 j+3\rangle}$,

$u_{i j}^{-}:=\frac{\langle 2 i, 2 j+2\rangle\langle 2 i+2,2 j\rangle}{\langle 2 i, 2 j\rangle\langle 2 i+2,2 j+2\rangle}$.

Applying a series of Plücker identities

$\langle i j\rangle\langle k l\rangle=\langle i k\rangle\langle j l\rangle+\langle i l\rangle\langle k j\rangle$

we can check the Y-system (evaluated at a fixed spectrum parameter $\zeta=0$ ) found in $[17,24]$ :

$$
\left(1-u_{i j+1}^{ \pm}\right)\left(1-u_{i+1 j}^{ \pm}\right)=\left(1-1 / u_{i j}^{ \pm}\right)\left(1-1 / u_{i+1 j+1}^{ \pm}\right),
$$

which constitutes two separate sets of equations keeping the sets of cross ratios $u^{+}$and $u^{-}$independent from each other.

\section{Cluster algebras and Stasheff polytopes}

The subject of cluster algebras was recently presented in [11], in a way accessible to physicists and complementing the standard references $[16,20,25,26]$. We review here basic concepts and terms.

We are only interested in the finite type cluster algebras. Such cluster algebras have a finite set of distinct generators (cluster variables), grouped in a finite number of clusters (sets) of equal size and that relate to each other by exchange relations where one of the cluster variables is replaced by (mutates to) another cluster variable outside the cluster. These exchange relations can be codified within each cluster by associating them with oriented quivers. By the quiver associated to a cluster we can define $\mathcal{X}$-coordinates related to each cluster variable in that cluster.

A cluster may contain a subset of frozen variables (or coefficients) that do not mutate and stay the same in all clusters. The number of cluster variables (not frozen ones) in a cluster is the rank of the cluster algebra. We call both cluster variables and cluster coefficients $\mathcal{A}$-coordinates.

Quivers are built with arrows connecting vertices. Such vertices in a quiver are identified with $\mathcal{A}$-coordinates, while the arrows define exchange relations among $\mathcal{A}$-coordinates and the $\mathcal{X}$-coordinates of each vertex in the quiver. Quivers are such that loops and two-cycles are not allowed. Loops are arrows that have an origin that is the same as the target and two-cycles are a pair of arrows with opposite directions connecting the same two vertices. When a two-cycle appears after a mutation, the arrows 'cancel each other' and disappear.

A mutation of a cluster variable in a cluster, or vertex $k$ in the corresponding quiver, transforms it to a new quiver according to the following operations: 
- for every pair of arrows $i \rightarrow k$ and $k \rightarrow j$, add a new arrow $i \rightarrow j$,

- reverse all arrows that target $\mathrm{k}$ or depart from $\mathrm{k}$,

- proceed with all two-cycle cancelation.

There is a theorem [20] that classifies all finite type cluster algebras according to simply laced Lie algebras. It states that given a finite type cluster algebra, their clusters have quivers that are mutations equivalent to a Dynkin diagram of a Lie algebra, via identification of the principal part of its quiver. The principal part of a quiver is the quiver without frozen variables and arrows to or from them.

There can be more than one arrow between two vertices and a number can be added on top of each arrow for cases of multiple arrows. A skew symmetric adjacency matrix $\left(b_{i j}\right)$ can be defined from the quiver, where

$b_{i j}=\# \operatorname{arrows}(i \rightarrow j)-\# \operatorname{arrows}(j \rightarrow i)$

A cluster variable in vertex $k, a_{k}$, mutates to $a_{k}^{\prime}$ according to the exchange relation:

$a_{k}^{\prime} a_{k}=\prod_{i \mid b_{k i}>0} a_{i}^{b_{k i}}+\prod_{i \mid b_{k i}<0} a_{i}^{-b_{k i}}$.

In a quiver, for every vertex corresponding to a cluster variable we define its $\mathcal{X}$-coordinate $x_{i}$ in terms of the $\mathcal{A}$ coordinates $a_{j}$,

$x_{i}=\prod_{j \neq i} a_{j}^{b_{i j}}$

We remark that when a mutation occurs in one vertex forming a new cluster, the adjacency matrix changes accordingly, and following (3.3) the new cluster will have different $\mathcal{X}$-coordinates. The $\mathcal{X}$-coordinate of vertex $i$ under mutation, mutates from $x_{i}$ to $x_{i}^{-1}$. Throughout the paper, we will not count a $\mathcal{X}$-coordinate and its inverse as independent $\mathcal{X}$ coordinates.

A useful construction associated to a finite type cluster algebra is a generalized associahedron. Such a construction represents the cluster algebra as a polytope with clusters being represented by vertices and mutations between clusters being represented by edges connecting vertices. For a rank $r$ cluster algebra, each vertex is parametrized by the $r \mathcal{X}$ coordinates of the represented cluster and from each vertex departs $r$ edges.

In a type A cluster algebra the generalized associahedron is called a Stasheff polytope [20]. This will be used from now on, since we will be always dealing with type A cluster algebras in special 2D kinematics.

Calling the rank of a Stasheff polytope the rank of the corresponding cluster algebra, an interesting property of a Stasheff polytope is that lower rank Stasheff polytopes cor- responding to cluster sub-algebras can easily be identified in the polytope.

Rank one $A_{1}$ and rank two $A_{1} \times A_{1}$ and $A_{2}$ cluster algebras are associated to the smallest Stasheff polytopes which are dimension-one edge and dimension-two quadrilateral and pentagonal faces, respectively.

\section{Configuration space and its cluster structure}

A configuration space is the space of parametrization of the amplitudes. In four dimensions, the configuration space has dimension $(3 n-15)$ as the space of external $n>4$ points in $\mathbb{P}^{3}$ (momentum twistors) modulo the action of the conformal group $P G L_{4}$ in $\mathbb{P}^{3}$. We denote it by $\operatorname{Conf}_{n}\left(\mathbb{P}^{3}\right)$. It can also be interpreted as the quotient of the space of $4 \times n$ matrices by the conformal group SL(4) and with each column quotient by $\mathbb{C}^{*}$ rescaling. The quotient of the space of $4 \times n$ matrices by the action of SL(4) and the diagonal subgroup $\mathbb{C}_{\text {diag }}^{*}$ of $\left(\mathbb{C}^{*}\right)^{n}$ (which rescales all columns by the same factor) is the Grassmannian $\operatorname{Gr}(4, n)$. Therefore,

$\operatorname{Conf}_{n}\left(\mathbb{P}^{3}\right) \cong \operatorname{Gr}(4, n) /\left(\mathbb{C}^{*}\right)^{n-1}$.

In the case of special 2D kinematics, momentum twistors (2.3) have one degree of freedom each and there are six conformal symmetries, which reduces the configuration space to $(n-6)$ dimensions.

As reviewed above, the cross ratios $u_{i j}^{ \pm}$depend only on either even or odd momentum twistors and they have separate equations, Eqs. (2.10), which leads us to expect that the configuration space might be divided into two subspaces of $(n / 2-3)$ dimensions each.

Momentum twistors contain only two nonzero components in special 2D kinematics. Considering their little group $\mathbb{C}^{*}$ each momentum twistor can be seen as a point in $\mathbb{P}^{1}$. Two separate $(2 \times n / 2)$ matrices can be constructed out of $n / 2$ points (even or odd indices only) in $\mathbb{P}^{1}$. They transform with the action of the conformal groups (in special 2D kinematics) $S L(2)_{+}$and $S L(2)_{-}$, respectively. Hence considering these two matrices modulo $S L(2)_{+} \times S L(2)_{-}$action and modulo a diagonal $\mathbb{C}_{\text {diag }}^{*}$ of each $\left(\mathbb{C}^{*}\right)^{n / 2}$, the configuration space is equivalent to two Grassmannians $\operatorname{Gr}(2, n / 2)_{+} \times \operatorname{Gr}(2, n / 2){ }_{-}$ of even and odd momentum twistors, respectively, each modulo the action of the remaining little group $\left(\mathbb{C}^{*}\right)^{n / 2-1}$ of the momentum twistors. We write it as $\operatorname{Conf}_{n / 2}\left(\mathbb{P}^{1}\right) \times$ $\operatorname{Conf}_{n / 2}\left(\mathbb{P}^{1}\right)$ and each copy $\operatorname{Conf}_{n / 2}\left(\mathbb{P}^{1}\right)$ has dimension $n / 2-3$.

Our remaining task is to study the cluster algebra of $\operatorname{Gr}(2, n / 2)$. The initial quiver $[25,26]$ can be drawn as follows: 


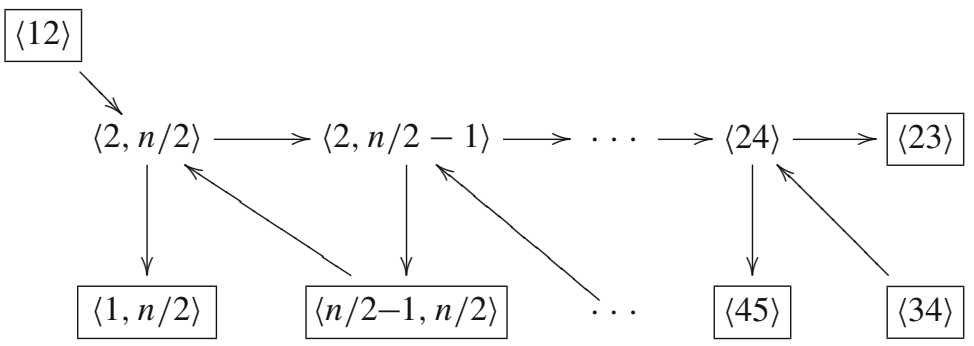

Here the numbers $\mathrm{i}$ in $\langle i \ldots\rangle$ are column positions in a general $\operatorname{Gr}(2, n / 2)$ and they correspond to the momentum twistor position index $2 \mathrm{i}$ in the $\operatorname{Gr}(2, n / 2)_{+}$and position index $2 \mathrm{i}-1$ in the $\operatorname{Gr}(2, n / 2)+$ case. For the rest of this section we will work on a general $\operatorname{Gr}(2, n / 2)$, except when mentioned otherwise.

This quiver has the form of the $A_{n / 2-3}$ Dynkin diagram, which classifies it as a finite cluster algebra of type $A_{n / 2-3}$ [20]. The rank of the Lie algebra corresponds to the number of cluster variables in a cluster, and it is equal to the dimensionality of $\operatorname{Conf}_{n / 2}\left(\mathbb{P}^{1}\right)$. The positive roots of the Lie algebra is in bijective correspondence with the non-initial cluster variables. In $A_{m}$, there are $m(m+1) / 2$ positive roots. Another important fact from $A_{m}$ cluster algebras is that the number of clusters is given by the number of triangulations of a polygon with $(m+3)$ sides and it is the $(m+1)$ th Catalan number $C_{m+1}$.

In summary, we have for an $A_{n / 2-3}$ cluster algebra:

- Its clusters (or quivers) contain $n / 2-3$ cluster variables each.

- The total number of cluster variables is equal the rank $(n / 2-3)$ plus the number of positive roots of $A_{n / 2-3}$ root system:

$$
\frac{(n / 2-3) n}{4}
$$

- The number of clusters is $C_{n / 2-2}=\frac{(n-4) !}{(n / 2-1) !(n / 2-2) !}$.

The $\mathcal{X}$-coordinates of the initial quiver can easily be worked out from (4.2). For $4 \leq j<n / 2$, the $\mathcal{X}$-coordinates corresponding to $\mathcal{A}$-coordinates $\langle 2, j\rangle$ is

$$
\begin{aligned}
& \frac{\langle 2, j-1\rangle\langle j, j+1\rangle}{\langle j-1, j\rangle\langle 2, j+1\rangle} \text { or }(\text { for } j=n / 2) \\
& \frac{\langle 1, n / 2\rangle\langle 2, n / 2-1\rangle}{\langle 12\rangle\langle n / 2-1, n / 2\rangle}
\end{aligned}
$$

We identify all cluster coordinates from a $A_{n / 2-3}$ cluster algebra, via a geometric interpretation of the cluster algebra as triangulations of a polygon $[20,26]$ in the following way.
- A cluster is associated to a triangulation of an $n / 2$-gon, such that no diagonal crosses any other.

- $\mathcal{A}$-coordinates $\langle i j\rangle$ (with $i<j$ ) of a cluster correspond to edges linking vertices $\mathrm{i}$ and $\mathrm{j}$ of the polygon. They are frozen variables when they correspond to side edges $(j=i+1)$ and cluster variables when in correspondence to diagonals $(j>i+1)$ of the polygon triangulation.

- A mutation is associated to changing one diagonal to another diagonal such that both are diagonals of the same quadrilateral (Fig. 1).

We can also identify the $\mathcal{X}$-coordinates of a given cluster by the associated polygon triangulation. For $i<k<j<l$, the diagonals of Fig. 1 represent two $\mathcal{A}$-coordinates $\langle i j\rangle$ and $\langle k l\rangle$; one is a mutation from another. Their $\mathcal{X}$-coordinates are given by the sides of the quadrilateral ikjl:

$x_{i j}=x_{k l}^{-1}=\frac{\langle i k\rangle\langle j l\rangle}{\langle k j\rangle\langle i l\rangle}$.

In order for $\mathcal{A}$-coordinates $\langle i j\rangle$ of a $\operatorname{Gr}(2, n / 2)_{ \pm}$cluster sub-algebra to be associated to the diagonals (and not the sides) in an $n / 2$-gon whose vertices are all even or all odd (Fig. 2), it is required that for $i<j$,

$4 \leq j-i \leq n-4$

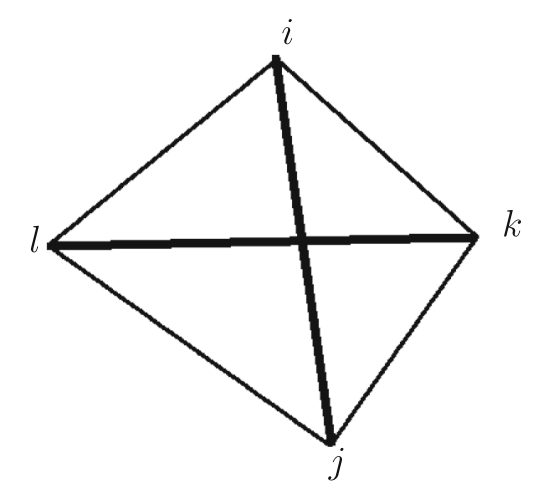

Fig. 1 Diagonals $\overline{i j}$ and $\overline{k l}$ define two possible triangulations for the quadrilateral $i k j l$ 

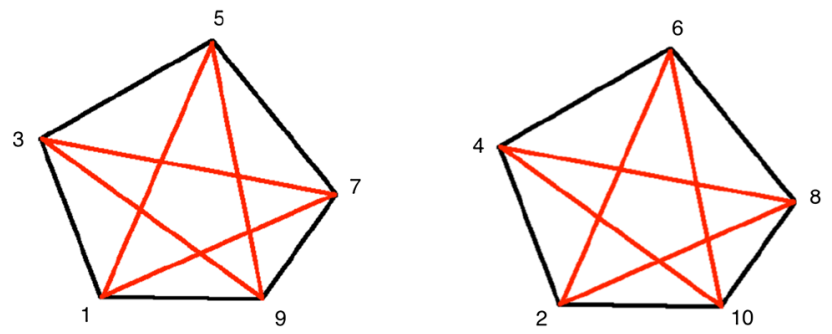

Fig. 2 Pentagons corresponding to an $A_{2} \times A_{2}$ cluster algebra for $n=$ 10 external particles in 2D kinematics. Their ten diagonals correspond to all cluster variables

\section{Results for $n=8,10$, and 12}

For such cases we are able to directly compare cluster coordinates with the cross ratios found in the two-loop MHV remainder functions expressed in [17]. These cases show how cross ratios fit as $\mathcal{X}$-coordinates. In the third case $(n=12)$ we observe a special selection of $\mathcal{X}$-coordinates, which we will explore further and generalize in the next section.

\section{$5.1 n=8$}

At $n=8$ the $A_{1} \times A_{1}$ cluster algebra is of rank 2 and there are two clusters for each $A_{1}$ cluster sub-algebra making a total of four cluster $\mathcal{A}$-coordinates. Equation (4.4) determines two initial $\mathcal{X}$-coordinates:

$x^{+}=\frac{\langle 1,7\rangle\langle 3,5\rangle}{\langle 1,3\rangle\langle 5,7\rangle}$ and $x^{-}=\frac{\langle 2,8\rangle\langle 4,6\rangle}{\langle 2,4\rangle\langle 6,8\rangle}$.

Using the nomenclature in (2.7) and (2.8), we find that

$$
\begin{aligned}
u_{15} & =u_{24}^{+}=\frac{x^{+}}{1+x^{+}}=\frac{1}{1+\frac{1}{x^{+}}}, \quad u_{26}=u_{13}^{-} \\
& =\frac{x^{-}}{1+x^{-}}=\frac{1}{1+\frac{1}{x^{-}}}, \\
u_{37} & =u_{13}^{+}=\frac{1}{1+x^{+}}, u_{48}=u_{24}^{-}=\frac{1}{1+x^{-}},
\end{aligned}
$$

From the above, $x^{+}$and $x^{-}$are the well-known cross ratios $\chi^{+}$and $\chi^{-}[17,22,27]$. The remaining cluster $\mathcal{X}$ coordinates are their respective cluster mutations $\left(x^{+}\right)^{-1}$ and $\left(x^{-}\right)^{-1}$. The standard cross ratios $u_{i j}$ are simple transformations of the cluster $\mathcal{X}$-coordinates, as remarked in [11,18]:

$u_{i}=\frac{1}{1+v_{i}}$

where $u_{i}$ is a cross ratio and $v_{i}$ is a $\mathcal{X}$-coordinate. Such a transformation preserves the intrinsic positivity of $\mathcal{X}$ coordinates, essential to keep cross ratios in a good kinematic domain. See [11] for a discussion of what a good kinematic domains is.

\section{$5.2 n=10$}

The corresponding $A_{2} \times A_{2}$ cluster algebra is of rank 4 and there are ten cluster variables and ten $\mathcal{X}$-coordinates. Equation (4.4) determines the four initial $\mathcal{X}$-coordinates:

$$
\left\{\frac{\langle 3,7\rangle\langle 1,9\rangle}{\langle 1,3\rangle\langle 7,9\rangle}, \frac{\langle 3,5\rangle\langle 7,9\rangle}{\langle 3,9\rangle\langle 5,7\rangle}\right\} \quad \text { and }\left\{\frac{\langle 4,8\rangle\langle 2,10\rangle}{\langle 2,4\rangle\langle 8,10\rangle}, \frac{\langle 4,6\rangle\langle 8,10\rangle}{\langle 4,10\rangle\langle 6,8\rangle}\right\}
$$

The remaining $\mathcal{X}$-coordinates can be retrieved by the following transitions of pentagon triangulations (Fig. 3), which represent cluster transitions by mutating one coordinate at a time:

$$
\begin{array}{ll}
\left\{\frac{\langle 1,3\rangle\langle 7,9\rangle}{\langle 3,7\rangle\langle 1,9\rangle}, \frac{\langle 1,7\rangle\langle 3,5\rangle}{\langle 1,3\rangle\langle 5,7\rangle}\right\}, & \left\{\frac{\langle 2,4\rangle\langle 8,10\rangle}{\langle 4,8\rangle\langle 2,10\rangle}, \frac{\langle 2,8\rangle\langle 4,6\rangle}{\langle 2,4\rangle\langle 6,8\rangle}\right\} \\
\left\{\frac{\langle 1,5\rangle\langle 7,9\rangle}{\langle 1,9\rangle\langle 5,7\rangle}, \frac{\langle 1,3\rangle\langle 5,7\rangle}{\langle 1,7\rangle\langle 3,5\rangle}\right\}, & \left\{\frac{\langle 2,6\rangle\langle 8,10\rangle}{\langle 2,10\rangle\langle 6,8\rangle}, \frac{\langle 2,4\rangle\langle 6,8\rangle}{\langle 2,8\rangle\langle 4,6\rangle}\right\} \\
\left\{\frac{\langle 1,9\rangle\langle 5,7\rangle}{\langle 1,5\rangle\langle 7,9\rangle}, \frac{\langle 1,3\rangle\langle 5,9\rangle}{\langle 1,9\rangle\langle 3,5\rangle}\right\}, & \left\{\frac{\langle 2,10\rangle\langle 6,8\rangle}{\langle 2,6\rangle\langle 8,10\rangle}, \frac{\langle 2,4\rangle\langle 6,10\rangle}{\langle 2,10\rangle\langle 4,6\rangle}\right\} \\
\left\{\frac{\langle 5,7\rangle\langle 3,9\rangle}{\langle 3,5\rangle\langle 7,9\rangle}, \frac{\langle 1,9\rangle\langle 3,5\rangle}{\langle 1,3\rangle\langle 5,9\rangle}\right\}, & \left\{\frac{\langle 6,8\rangle\langle 4,10\rangle}{\langle 4,6\rangle\langle 8,10\rangle}, \frac{\langle 2,10\rangle\langle 4,6\rangle}{\langle 2,4\rangle\langle 6,10\rangle}\right\}
\end{array}
$$

Avoiding to choose both $x$ and $x^{-1}$, our $10 \mathcal{X}$-coordinates are:

$$
\begin{aligned}
& v_{1}=\frac{\langle 13\rangle\langle 57\rangle}{\langle 17\rangle\langle 35\rangle}, \quad v_{3}=\frac{\langle 35\rangle\langle 79\rangle}{\langle 39\rangle\langle 57\rangle}, \quad v_{5}=\frac{\langle 19\rangle\langle 57\rangle}{\langle 15\rangle\langle 79\rangle}, \\
& v_{7}=\frac{\langle 13\rangle\langle 79\rangle}{\langle 37\rangle\langle 19\rangle}, \quad v_{9}=\frac{\langle 19\rangle\langle 35\rangle}{\langle 13\rangle\langle 59\rangle} \\
& v_{2}=\frac{\langle 24\rangle\langle 68\rangle}{\langle 28\rangle\langle 46\rangle}, \quad v_{4}=\frac{\langle 46\rangle\langle 8,10\rangle}{\langle 4,10\rangle\langle 68\rangle}, \quad v_{6}=\frac{\langle 2,10\rangle\langle 6,8\rangle}{\langle 2,6\rangle\langle 8,10\rangle}, \\
& v_{8}=\frac{\langle 24\rangle\langle 8,10\rangle}{\langle 48\rangle\langle 2,10\rangle}, \quad v_{10}=\frac{\langle 2,10\rangle\langle 4,6\rangle}{\langle 2,4\rangle\langle 6,10\rangle} .
\end{aligned}
$$

Turning back to cross ratios, the condition (4.5) only allows the nontrivial cross ratios $u_{i, i+4}$ for $i=1, \ldots, 10$ with indices taken modulo 10. Denoting $u_{i}=u_{i, i+4}$ (as in [17]), we find again that all nontrivial cross ratios are the same simple functions, see Eq. (5.4), of the cluster $\mathcal{X}$-coordinates.

$5.3 n=12$

The $A_{3} \times A_{3}$ cluster algebra is of rank 6 and there are 18 cluster variables (4.3). Each $A_{3}$ sub-algebra contains $C_{4}=$ 14 clusters (number of triangulations of an hexagon), and it would be cumbersome to show all of them here in order to calculate their $\mathcal{X}$-coordinates. Hence, we appeal to [11], which found $15 \mathcal{X}$-coordinates for an $A_{3}$ cluster algebra. Here we notice the remarkable mismatch between the number of cross ratios (18) used in the $n=12$ two-loop remainder function in [17] and the $\mathcal{X}$-coordinates (30). Similarly, in [11], the authors found that at $n=6$, in $4 \mathrm{D}$ kinematics, only nine out of $15 \mathcal{X}$-coordinates of a single $A_{3}$ cluster algebra were related to the cross ratios of the motivic amplitudes. 


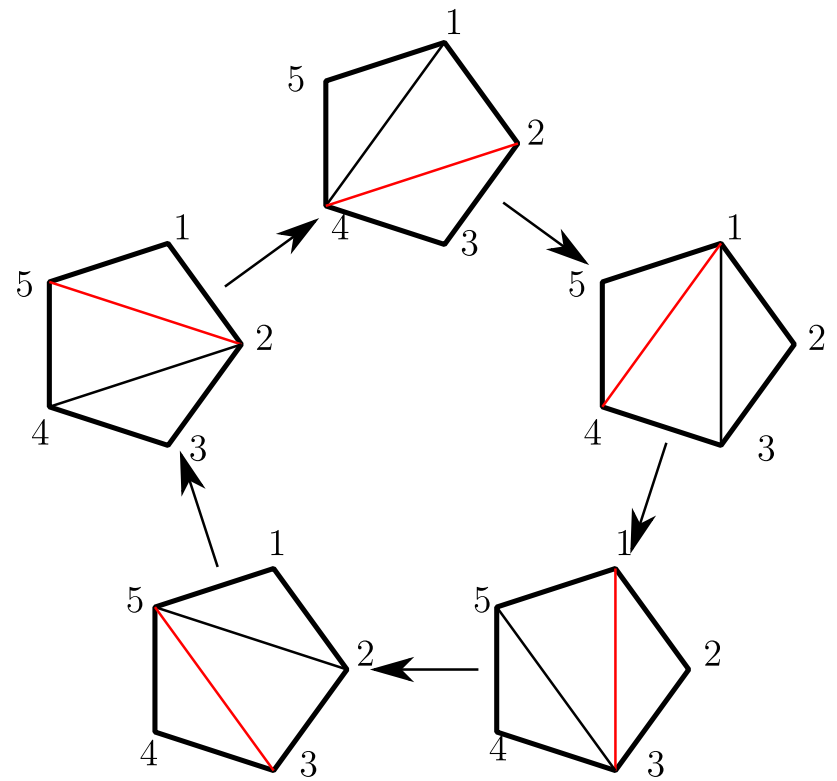

Fig. 3 Transition between pentagon triangulations representing transition between different clusters via a mutation of one of its coordinates (red diagonal)

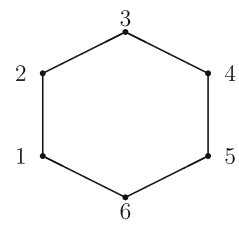

(a)

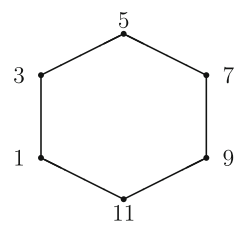

(b)

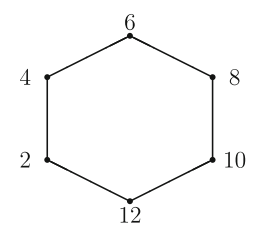

(c)
Fig. 4 In 4D kinematics, $n=6, \mathcal{X}$-coordinates are obtained from triangulation of the hexagon (a). In 2D kinematics, $n=12$, the $\mathcal{X}$ coordinates are obtained from triangulations of hexagons $\mathbf{b}$ and $\mathbf{c}$

These $\mathcal{X}$-coordinates can be obtained from triangulations of the hexagon (Fig. 4a). We report below the nine selected $\mathcal{X}$ coordinates found in [11]:

$$
\begin{array}{lll}
\frac{\langle 23\rangle\langle 56\rangle}{\langle 35\rangle\langle 26\rangle}, & \frac{\langle 61\rangle\langle 34\rangle}{\langle 13\rangle\langle 64\rangle}, & \frac{\langle 45\rangle\langle 12\rangle}{\langle 15\rangle\langle 24\rangle}, \\
\frac{\langle 12\rangle\langle 34\rangle}{\langle 14\rangle\langle 23\rangle}, & \frac{\langle 56\rangle\langle 12\rangle}{\langle 25\rangle\langle 16\rangle}, & \frac{\langle 34\rangle\langle 56\rangle}{\langle 36\rangle\langle 45\rangle}, \\
\frac{\langle 45\rangle\langle 16\rangle}{\langle 14\rangle\langle 56\rangle}, & \frac{\langle 23\rangle\langle 45\rangle}{\langle 25\rangle\langle 34\rangle}, & \frac{\langle 61\rangle\langle 23\rangle}{\langle 63\rangle\langle 12\rangle}
\end{array}
$$

We made above a convenient choice between some $\mathcal{X}$ coordinates and their inverse, which will become clear later. Will the $\mathcal{X}$-coordinates selected above also play a role in special 2D kinematics? At $n=12$ in special 2D kinematics, we have two copies of the $A_{3}$ cluster algebra and therefore two copies of the above $\mathcal{X}$-coordinates, one with all odd particles and the other with all even particles (Fig. 4b, c). They are:

$$
\begin{array}{rrrr}
v_{5,11}=\frac{\langle 57\rangle\langle 11,1\rangle}{\langle 7,11\rangle\langle 51\rangle} & v_{17}=\frac{\langle 13\rangle\langle 79\rangle}{\langle 37\rangle\langle 19\rangle}, & v_{39}=\frac{\langle 35\rangle\langle 9,11\rangle}{\langle 3,11\rangle\langle 59\rangle} \\
v_{4,10}=\frac{\langle 4,6\rangle\langle 10,12\rangle}{\langle 6,10\rangle\langle 4,12\rangle} & v_{6,12}=\frac{\langle 12,2\rangle\langle 68\rangle}{\langle 26\rangle\langle 12,8\rangle} & v_{2,8}=\frac{\langle 24\rangle\langle 8,10\rangle}{\langle 4,10\rangle\langle 48\rangle} \\
v_{37}=\frac{\langle 35\rangle\langle 79\rangle}{\langle 39\rangle\langle 57\rangle} & v_{11,3}=\frac{\langle 11,1\rangle\langle 35\rangle}{\langle 5,11\rangle\langle 31\rangle} & v_{7,11}=\frac{\langle 79\rangle\langle 11,1\rangle}{\langle 71\rangle\langle 9,11\rangle} \\
v_{26}=\frac{\langle 24\rangle\langle 68\rangle}{\langle 28\rangle\langle 46\rangle} & v_{10,2}=\frac{\langle 10,12\rangle\langle 24\rangle}{\langle 4,10\rangle\langle 2,12\rangle} & v_{6,10}=\frac{\langle 68\rangle\langle 10,12\rangle}{\langle 6,12\rangle\langle 8,10\rangle} \\
v_{91}=\frac{\langle 9,11\rangle\langle 13\rangle}{\langle 39\rangle\langle 1,11\rangle} & v_{59}=\frac{\langle 57\rangle\langle 9,11\rangle}{\langle 5,11\rangle\langle 79\rangle} & v_{15}=\frac{\langle 13\rangle\langle 57\rangle}{\langle 17\rangle\langle 35\rangle} \\
v_{8,12}=\frac{\langle 8,10\rangle\langle 2,12\rangle}{\langle 28\rangle\langle 10,12\rangle} & v_{48}=\frac{\langle 46\rangle\langle 8,10\rangle}{\langle 4,10\rangle\langle 68\rangle} & v_{12,4}=\frac{\langle 12,2\rangle\langle 46\rangle}{\langle 12,6\rangle\langle 24\rangle} .
\end{array}
$$

Once more we find the same direct relation of the above $\mathcal{X}$ coordinates to the preferred set of cross ratios (5.4) chosen in [17] for describing $\mathcal{R}_{12}^{(2)}$ in special 2D kinematics. Rewriting the expression (2.7) for cross ratios using the Plücker relation:

$u_{i j}=\frac{1}{1+\frac{\langle i, i+2\rangle\langle j, j+2\rangle}{\langle i, j+2\rangle\langle i+2, j\rangle},}$

which implies that the 18 nontrivial cross ratios in [17] for $n=12, u_{i, i+4}$, and $u_{i, i+6}, i=1, \ldots, 12$ (cyclic indices), are simple expressions (5.4) of the above $\mathcal{X}$-coordinates of the $A_{3} \times A_{3}$ cluster algebra:

$u_{i, i+4}=\frac{1}{1+v_{i, i+4}} \quad$ and $\quad u_{i, i+6}=\frac{1}{1+v_{i, i+6}}$

for $i=1, \ldots, 12$ and indices modulo 12 .

Intriguingly, $12 \mathcal{X}$-coordinates made no appearance in the relation to the cross ratios, which led us to take a more careful look at the $18 \mathcal{X}$-coordinates that appeared in Eq. (5.11). For simplicity, we will work with only one copy of the $A_{3}$ of $A_{3} \times A_{3}$ cluster algebra.

We start by noticing that $\mathcal{X}$-coordinates in (5.9) originate from clusters associated to snake triangulations. According to the expression (4.5), looking at Fig. 5, we see that the $\mathcal{X}$-coordinates corresponding to diagonals in the snake triangulations (a), (b), and (c) are

$\begin{array}{lll}\text { hexagon (a) } & \text { hexagon (b) } & \text { hexagon (c) } \\ \overline{17} \rightarrow v_{17} & \overline{39} \rightarrow v_{1,7}^{-1} & \overline{17} \rightarrow v_{5,11}^{-1} \\ \overline{19} \rightarrow v_{7,11}^{-1} & \overline{19} \rightarrow v_{19} & \overline{711} \rightarrow v_{7,11} \\ \overline{37} \rightarrow v_{15}^{-1} & \overline{37} \rightarrow v_{37} & \overline{15} \rightarrow v_{15}\end{array}$

Continuing by checking with the three other remaining snake triangulations (not shown here) or simply rotating the indices, one can realize that all nine $\mathcal{X}$-coordinates of odd parity that are directly related to the cross ratios are part of the clusters associated to snake triangulations. We will call them snake clusters.

Notice that these snake triangulations have the remarkable property of projecting the $A_{1}$ and $A_{1} \times A_{1}$ cluster subalgebras out of the $A_{3}$ cluster algebra. These cluster sub- 


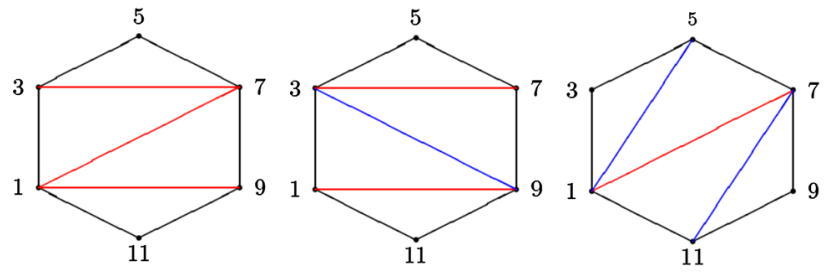

Fig. 5 Three out of six snake triangulations in the hexagon. The triangulation (b) is a mutation exchange of diagonal $\overline{17}$ in a and the triangulation (c) is two mutations away from a by exchanging step diagonals $\overline{37}$ and $\overline{19}$ by $\overline{15}$ and $\overline{711}$, respectively

algebras correspond to lines and quadrilateral faces in the $A_{3}$ Stasheff polytope [11] passing through six snake clusters vertices in the equator of the polytope. We see that by noticing in Fig. 5 that the snake triangulation in (a) mutates to the snake triangulation in (b) by exchanging diagonal $\overline{17}$ to $\overline{39}$, which exchanges $v_{17}$ by its inverse. But in order to change from the snake triangulation in (a) to (c) two mutations are necessary, and these two mutations can be done in any order, characterizing a $A_{1} \times A_{1}$ quadrilateral face:

$$
\begin{aligned}
v_{i j}= & \frac{\langle i, i+2\rangle\langle j, j+2\rangle}{\langle i, j+2\rangle\langle i+2, j\rangle}, \quad 4 \leq j-i \leq n-4, \\
& (j-i) \text { even. }
\end{aligned}
$$

We saw before that for $n=12$ there is a larger number of $\mathcal{X}$-coordinates than standard cross ratios and that some $\mathcal{X}$ coordinates are, like (6.1), directly related to the cross ratios (2.7), setting them apart from the remaining $\mathcal{X}$-coordinates. We show below that the cluster structure offers some explanation, highlighting them in the cluster structure.

Most of the concepts have already been presented in Sect. 5.3. Here we will put it in general terms. Again, for simplification, we will look only at one $A_{n / 2-3}$ cluster subalgebra of the entire $A_{n / 2-3} \times A_{n / 2-3}$ cluster algebra.

In order to show (6.1), we start by noticing that any $\overline{i j}$ diagonal can be part of a zig-zag or snake triangulation [20] of a polygon of $n / 2$ sides. From a initial snake triangulation, all other snake triangulations can be obtained by a step by step mutation exchange of diagonals without common vertices in the initial triangulation (Fig. 8) (mutating only the zig and skipping the zag diagonals). In this way, any diagonal $\overline{i j}$ is

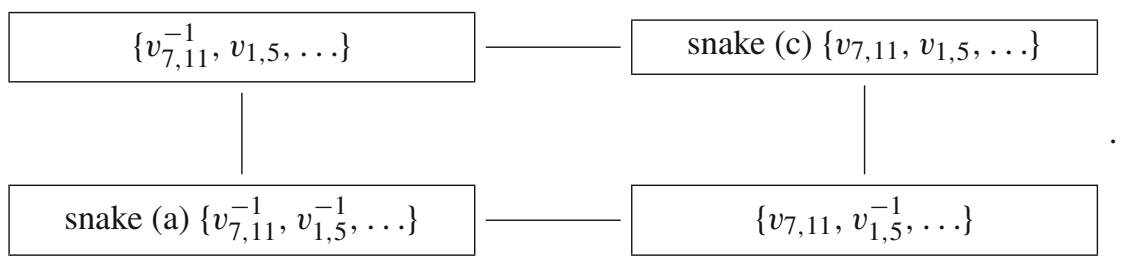

The transitions between snake triangulations (a) and (c) can be seen as the freezing of the diagonal $\overline{17}$, leading to the formation of two quadrilaterals $\overline{1357}$ and $\overline{79111}$ each representing an $A_{1}$ cluster sub-algebra. The $A_{1} \times A_{1}$ cluster algebra has only two $\mathcal{X}$-coordinates and in diagram (5.13) they are $v_{7,11}$ and $v_{1,5}$.

Figure 6 shows the partial Stasheff polytope obtained by transitioning between snake triangulations (or snake clusters). Each vertex represents a cluster defined by its three $\mathcal{X}$-coordinates. Edges connecting vertices represent a mutation exchange between the clusters. The vertices outside the equator do not correspond to snake triangulations and they are parametrized by some of the six $\left\{e_{i}\right\} \mathcal{X}$-coordinates not mentioned in (5.9).

\section{General $n$ external particles}

Expression (5.10) suggest us that the standard basis of cross ratios $u_{i j}$ ( $i$ and $\mathrm{j}$ are of same parity) in special 2D kinematics (2.7) are an expression of the $\mathcal{X}$-coordinates $v_{i j}$ of the form $u_{i j}^{-1}=1+v_{i j}$, once we are able to show that there is a set of $\mathcal{X}$-coordinates given by part of a unique 'snake' triangulation, crossing quadrilateral $\{i, i+2, j, j+2\}$ (Fig. 7). The $\mathcal{X}$-coordinates of a diagonal $\overline{i j}$ in such a snake triangulation are of the form (6.1). The total number of such $\mathcal{X}$-coordinates is the same as the total number of $\mathcal{A}$-coordinates and the number of cross ratios (2.7).

The total number of snake triangulations of an $n / 2$-gon is $n / 2$. In the corresponding Stasheff polytope, these $n / 2$ vertices will be separated by hypercubes of dimension $p=$ $\left[\frac{n / 2-3}{2}\right]$ and $q=n / 2-3-p$, where brackets mean the integer part. These hypercubes represent $A_{1} \times A_{1} \times \cdots A_{1}$ cluster sub-algebras of the $A_{n / 2-3}$ cluster algebra. These cluster sub-algebras $A_{1}^{p}$ and $A_{1}^{q}$ can be seen through mutations from one snake triangulation vertex to the next snake triangulation vertex in the Stasheff polytope. Departing from a snake triangulation (Fig. 8), the two simplest sets of mutations to get to another snake configuration are mutating all zig or all zag diagonals while keeping the remaining zag or zig diagonals unchanged. Keeping the diagonals unchanged can be seen as freezing of the $\mathcal{A}$-coordinates that they represent, and it leads to the splitting of the polygon in several quadrilaterals, each one corresponding to an $A_{1}$ cluster sub-algebra. In Fig. 9, we exemplify an $n=14$ case corresponding to a partial $A_{4}$ Stasheff polytope made of seven snake cluster ver- 


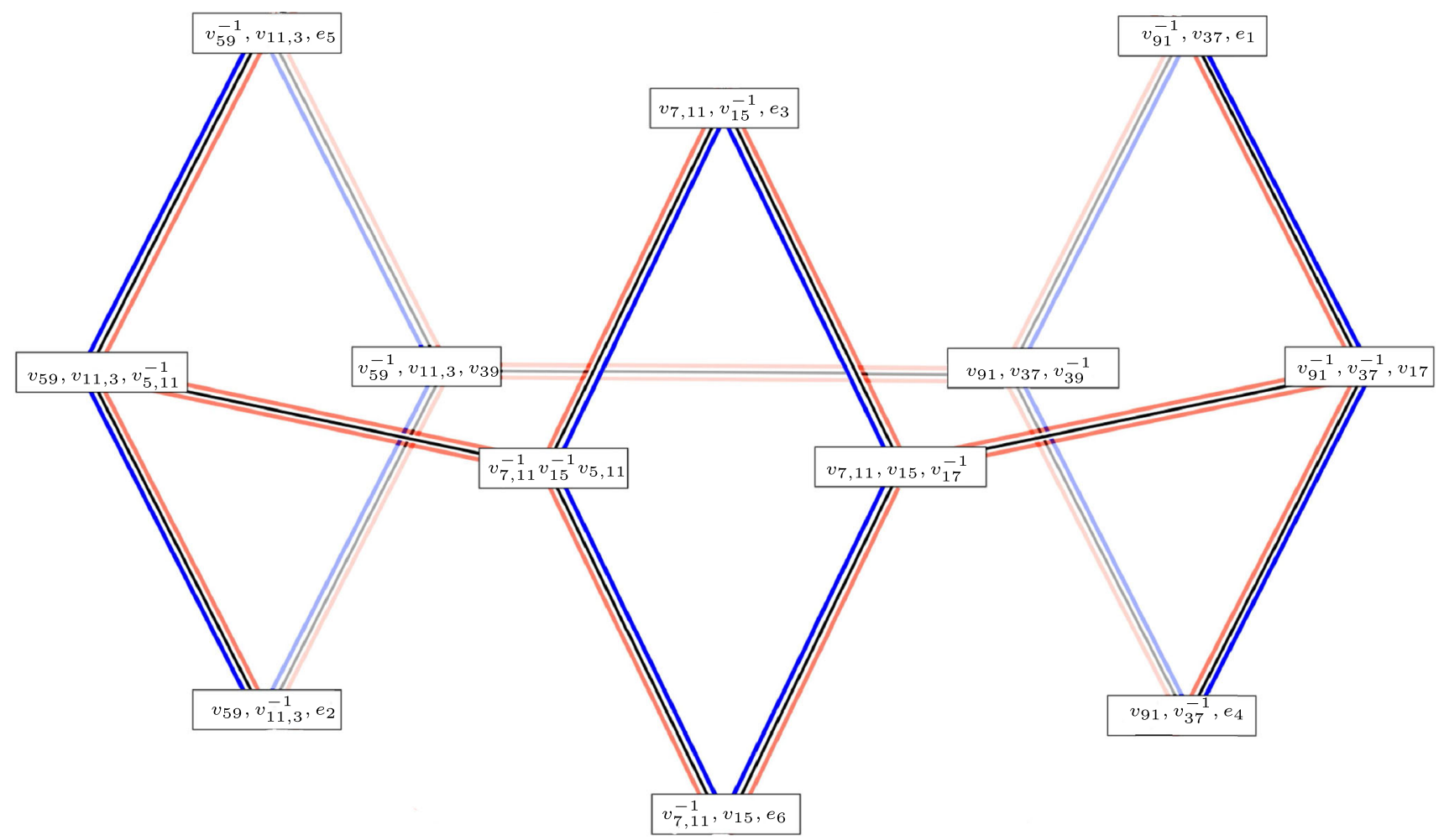

Fig. 6 Part of the Stasheff polytope from the $A_{3}$ cluster algebra, obtained by mutation exchanges between the six snake clusters vertices in the equator of the picture

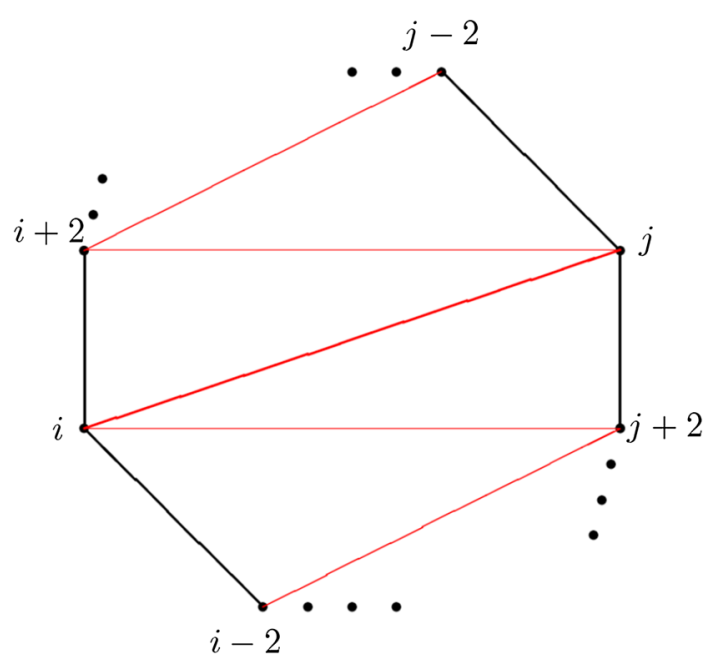

Fig. $7 \overline{i j}$ diagonal in a 'snake' triangulation of an n-gon

tices and seven quadrilaterals $(p=q=2)$. Such a structure of snake clusters and hypercubes in the Stasheff polytope we call a necklace of hypercube beads, or hypercube necklace, as Fig. 9 suggests. Freezing of different diagonals in the snake triangulation of an $n / 2$-gon generates other type A cluster sub-algebras and other geometrical pictures with one snake cluster vertex in the Stasheff polytope.

Hypercube necklaces in Stasheff polytopes are important in counting quadrilateral faces composed of the $\mathcal{X}$ coordinates from the snake clusters selected by the ampli-

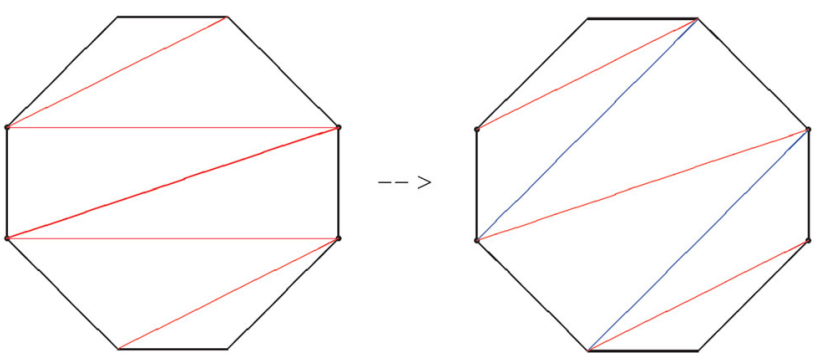

Fig. 8 Exchanging "snake" triangulations by two mutations of alternating "zig" diagonals

tude. Quadrilateral faces $\left(A_{1} \times A_{1}\right.$ cluster sub-algebra $)$ of the amplitude selected $\mathcal{X}$-coordinates were found [18] to be related to obstruction terms of the Bloch groups $B_{2} \times B_{2}$, which do not allow the MHV two-loop motivic amplitude to be written in terms of the sum of classical 4-logarithms. In 4D kinematics, no obstructions were found at $n=6\left(A_{3}\right.$ cluster algebra), but at $n=7$ ( $E_{6}$ cluster algebra) it was found that the obstruction is nontrivial and depends on only 42 quadrilateral faces, out of 1,785 existent in the $E_{6}$ Stasheff polytope.

\section{Conclusion}

Special 2D kinematics offers an interesting arena to study the cluster structure of the configuration space of a n-particle 


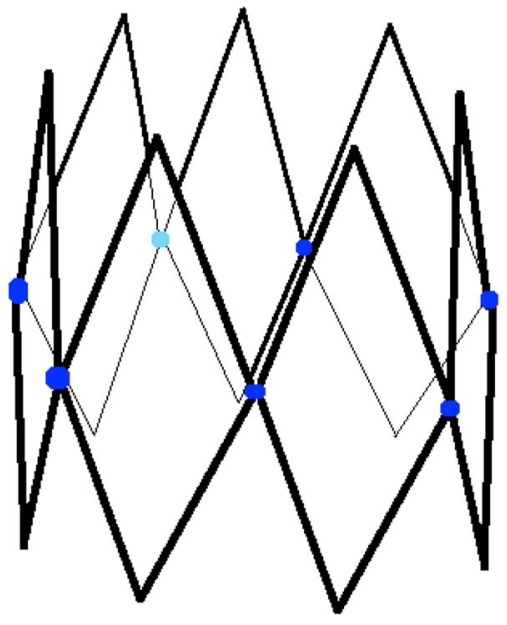

Fig. $9 A_{4}$ partial Stasheff polytope related to snake triangulations of an heptagon. Vertices in blue correspond to snake clusters. Seven quadrilaterals correspond to the $A_{1} \times A_{1}$ cluster sub-algebra

amplitude. Following the steps of [11], we notice the existence of a preferential choice of kinematic variables (cross ratios) that preserve the cluster structure of the configuration space of external particles and simultaneously lead to simple amplitude expressions ([17]) in special 2D kinematics. The cluster algebras in 2D kinematics are of $A_{i}$ type. $A_{i}$ cluster algebras also appear in 4D kinematics either as an entire cluster algebra or as cluster sub-algebras, and their importance remains in relation to the choice of nontrivial cross ratios.

In the present work, we showed the existence of special $\mathcal{X}$-coordinates among all $\mathcal{X}$-coordinates of a cluster algebra of type $A_{n}$. They are $\mathcal{X}$-coordinates of snake clusters, those whose associated polygon triangulation are snakes. In an $A_{n}$ cluster algebra, there are $n+3$ snake clusters. In a Stasheff polytope these snake clusters are opposite vertices of hypercubes of dimension $\mathrm{p}$ and $\mathrm{q}$ (a line if $p=1$ or quadrilateral face if $p=2$ ), such that $p=[n / 2]$ and $p+q=n$. These snake cluster vertices connected by hypercubes form a necklace in the $A_{n}$ Stasheff polytope with hypercubes as beads. We call it a hypercube necklace. Such a structure contains many $A_{1} \times A_{1}$ quadrilateral faces made of pairs of snake cluster $\mathcal{X}$-coordinates that can be important in relation to the obstruction terms found in [11]. For the $E_{6}$ cluster algebra, by freezing the cluster variables in the $E_{6}$ quiver we checked the presence of four $A_{4}$ necklaces and one $A_{5}$ necklace, summing altogether 56 quadrilateral faces, which some may play a special role as arguments in the $B_{2} \times B_{2}$ obstruction terms found at $n=7$ (4D kinematics) amplitude.

Furthermore, we showed that in special 2D kinematics, at any number of external particles, the canonical choice of cross ratios (2.7) selected by the simple two-loop MHV amplitude expression of [17] is directly related to these special $\mathcal{X}$-coordinates.
In [11], the authors noticed that in the two-loop MHV $n=6$ amplitude, only nine out of $15 \mathcal{X}$-coordinates appear in the remainder function $\mathcal{R}_{6}^{(2)}$. This case is the $A_{3}$ cluster algebra of $\operatorname{Conf}_{6}\left(\mathbb{P}^{3}\right)$. In special 2D kinematics the $A_{3}$ cluster algebra appears in the $n=12$ amplitude, and we showed that the same nine $\mathcal{X}$-coordinates out of 15 are selected to appear in the respective two-loop remainder function. The structure of the cluster algebra discussed here has a fundamental role to play in this mysterious selection. The fact that the same selection took place at 2D and 4D kinematics with different amplitude expressions reveals the importance of the cluster structure of the configuration space and its preservation at two loops.

The structures that we unlocked at special 2D kinematics are always of the $A_{n}$ type cluster algebra but once $A_{n}$ type cluster sub-algebras are part of the cluster structure of every configuration space, and other kinematical limits can lead to the appearance of the $A_{n}$ cluster algebra, we believe that this work program may have significant implications in the $4 \mathrm{D}$ kinematics scattering amplitudes.

Acknowledgments The author is indebted to Ruth Britto for stimulating discussions and criticism on the project and Bo Feng for early discussions on cluster algebra and his encouraging comments in the early stages of the project. The author also would like to thank Gregory Korchemsky, Simon Caron-Huot and Song He for helpful comments. The author is supported by CAPES (Brazilian research agency) postdoctoral fellowship.

Open Access This article is distributed under the terms of the Creative Commons Attribution License which permits any use, distribution, and reproduction in any medium, provided the original author(s) and the source are credited.

Funded by $\mathrm{SCOAP}^{3}$ / License Version CC BY 4.0.

\section{References}

1. J.M. Drummond, J. Henn, V.A. Smirnov, E. Sokatchev, JHEP 0701, 064 (2007). [arXiv:hep-th/0607160]

2. L.F. Alday, J. Maldacena, Gluon scattering amplitudes at strong coupling. JHEP 0706, 064 (2007). [arXiv:0705.0303 [hep-th]]

3. J.M. Drummond, G.P. Korchemsky, E. Sokatchev, Conformal properties of four-gluon planar amplitudes and Wilson loops. Nucl. Phys. B 795, 385 (2008). [arXiv:0707.0243 [hep-th]]

4. A. Brandhuber, P. Heslop, G. Travaglini, MHV amplitudes in N = 4 super Yang-Mills and Wilson loops. Nucl. Phys. B 794, 231 (2008). [arXiv:0707.1153 [hep-th]]

5. J.M. Drummond, J. Henn, G.P. Korchemsky, E. Sokatchev, On planar gluon amplitudes/Wilson loops duality. Nucl. Phys. B 795, 52 (2008). [arXiv:0709.2368 [hep-th]]

6. J.M. Drummond, J. Henn, G.P. Korchemsky, E. Sokatchev, Conformal ward identities for Wilson loops and a test of the duality with gluon amplitudes. Nucl. Phys. B 826, 337 (2010). [arXiv:0712. 1223 [hep-th]]

7. Z. Bern, L.J. Dixon, D.A. Kosower, R. Roiban, M. Spradlin, C. Vergu, A. Volovich, The Two-Loop Six-Gluon MHV Amplitude in Maximally Supersymmetric Yang-Mills Theory. Phys. Rev. D 78, 045007 (2008). [arXiv:0803.1465 [hep-th]] 
8. J.M. Drummond, J. Henn, G.P. Korchemsky, E. Sokatchev, Hexagon Wilson loop $=$ six-gluon MHV amplitude. Nucl. Phys. B 815, 142 (2009). [arXiv:0803.1466 [hep-th]]

9. C. Anastasiou, A. Brandhuber, P. Heslop, V. V. Khoze, B. Spence, G. Travaglini, Two-loop polygon wilson loops in N=4 SYM. JHEP 0905, 115 (2009). [arXiv:0902.2245 [hep-th]]

10. A.B. Goncharov, M. Spradlin, C. Vergu, A. Volovich, Classical polylogarithms for amplitudes and Wilson loops. Phys. Rev. Lett. 105, 151605 (2010). [arXiv:1006.5703 [hep-th]]

11. J. Golden, A.B. Goncharov, M. Spradlin, C. Vergu, A. Volovich, Motivic amplitudes and cluster coordinates. [arXiv:1305.1617 [hep-th]]

12. V. Del Duca, C. Duhr, V.A. Smirnov, An analytic result for the two-loop hexagon Wilson loop in N = 4 SYM. JHEP 1003, 099 (2010). [arXiv:0911.5332 [hep-ph]]

13. V. Del Duca, C. Duhr, V.A. Smirnov, The two-loop hexagon Wilson loop in N = 4 SYM. JHEP 1005, 084 (2010). [arXiv:1003.1702 [hep-th]]

14. J.-H. Zhang, On the two-loop hexagon Wilson loop remainder function in N=4 SYM. Phys. Lett. B 697, 370 (2011). [arXiv:1004.1606 [hep-th]]

15. M. Gekhtman, M. Shapiro, A. Vainshtein, Cluster algebras and Poisson geometry. [arXiv:math.QA/0208033]

16. S. Fomin, A. Zelevinsky, Cluster algebras I: foundations. J. Am. Math. Soc. 15(2), 497-529 (2002). [arXiv:math/0104151]

17. P. Heslop, V.V. Khoze, Analytic results for MHV Wilson loops. JHEP 1011, 035 (2010). [arXiv:1007.1805 [hep-th]]

18. J. Golden, M. Spradlin, JHEP 1309, 111 (2013). [arXiv:1306.1833 [hep-th]]
19. S. Caron-Huot, S. He, JHEP 1308, 101 (2013). [arXiv:1305.2781 [hep-th]]

20. S. Fomin, A. Zelevinsky, Cluster algebras. II: finite type classification. Invent. Math. 154(1), 63-121 (2003). [arXiv:math/0208229]

21. T. Goddard, P. Heslop, V.V. Khoze, Uplifting amplitudes in special kinematics. JHEP 1210, 041 (2012). [arXiv:1205.3448 [hep-th]]

22. L.F. Alday, J. Maldacena, Null polygonal Wilson loops and minimal surfaces in Anti-de-Sitter space. JHEP 0911, 082 (2009). [arXiv:0904.0663 [hep-th]]

23. N. Arkani-Hamed, J.L. Bourjaily, F. Cachazo, J. Trnka, Local integrals for planar scattering amplitudes. JHEP 1206, 125 (2012). [arXiv:1012.6032 [hep-th]]

24. L.F. Alday, D. Gaiotto, J. Maldacena, Thermodynamic Bubble Ansatz. JHEP 1109, 032 (2011). [arXiv:0911.4708 [hep-th]]

25. M. Gekhtman, M. Shapiro, A. Vainshtein, Cluster algebras and Poisson geometry. Mosc. Math. J. 3(3), 899-934 (2003)

26. B. Keller, Cluster algebras, quiver representations and triangulated categories. in Triangulated Categories. (Cambridge University Press, Cambridge, 2010). [arXiv:0807.1960 [math.RT]]

27. A. Brandhuber, P. Heslop, V.V. Khoze, G. Travaglini, Simplicity of polygon Wilson loops in N=4 SYM. JHEP 1001, 050 (2010). [arXiv:0910.4898 [hep-th]] 